\title{
Task-Oriented Display of Landmark Pictograms in Maps
}

\author{
Julian Keil ${ }^{a}, *$, Dennis Edler ${ }^{a}$, Lars Kuchinke ${ }^{b}$, Frank Dickmann ${ }^{a}$ \\ ${ }^{a}$ Ruhr University Bochum, Geography Department, Cartography, Bochum, Germany, julian.keil@rub.de, dennis.edler@rub.de, \\ frank.dickmann@rub.de \\ ${ }^{b}$ International Psychoanalytic University, Methodology and Evaluation, Berlin, Germany, lars.kuchinke@ipu-berlin.de \\ * Corresponding author
}

Keywords: Landmarks, Navigation, Route-Memory, Cognitive Cartography, Eye-Tracking

\begin{abstract}
:
When people use maps to plan and memorize a route, they commonly look for landmark representations in the form of pictograms that can be used as reference points for orientation (Anacta et al., 2017; Bestgen et al., 2016; Steck \& Mallot, 2000). During navigation, matching the positions of landmark pictograms with the positions of the represented objects allows them to verify that they are following the route correctly (Golledge, 1999). When route planning functions of web mapping services as OpenStreetMap or Google Maps are used, the width of the map margins around the displayed route determines which, and how many, landmark representations are displayed in the map. Therefore, margin widths should be selected based on the relevance of specific landmarks around the route for memorizing and following the route. However, this requires to identify task-relevant landmark representations first.

In order to identify factors predicting the task-relevance of landmark pictograms, we carried out two route recognition experiments on an eye-tracker monitor. These experiments examined how the relative position of landmark pictograms and the visual complexity of the map affect gaze pattern when a route is memorized. We hypothesized that landmark pictograms close to the route, decision points and potential decision points would receive more visual attention, as they can act as local reference points. Additionally, we expected that more distant landmark pictograms receive visual attention if the visual complexity of the map is low and therefore less reference points for memorizing the route are available.

The results demonstrate that the relative position of a landmark pictograms is indeed a relevant predictor for visual attention. The closer landmark pictograms were to the route, decision points or potential decision points, the longer they were fixated. As expected, the average distance of all fixated landmark pictograms was also higher in maps with a low visual complexity. We conclude that small map margins around the route may be applied if a map has a high visual complexity, as enough reference points are available in the close surroundings of the route. Additionally, reducing map margins would increase the presentation size of the remaining map elements and thereby their readability. However, in maps with a low visual complexity, e.g. rural areas, large margins around the route should be applied to provide a sufficient amount of visual reference points, as landmark pictograms, for memorizing the route.
\end{abstract}

